\title{
\begin{tabular}{l|l} 
Mibraries & DSpace@MIT
\end{tabular}
}

\author{
MIT Open Access Articles
}

\begin{abstract}
Wake structure and sediment deposition behind models of submerged vegetation with and without flexible leaves
\end{abstract}

The MIT Faculty has made this article openly available. Please share how this access benefits you. Your story matters.

Citation: Zhenghong, Hu et al. "Wake structure and sediment deposition behind models of submerged vegetation with and without flexible leaves." Advances in Water Resources 118 (c) 2018 Elsevier Ltd

As Published: http://dx.doi.org/10.1016/j.advwatres.2018.06.001

Publisher: Elsevier BV

Persistent URL: https://hdl.handle.net/1721.1/123897

Version: Author's final manuscript: final author's manuscript post peer review, without publisher's formatting or copy editing

Terms of use: Creative Commons Attribution-NonCommercial-NoDerivs License 


\section{Wake structure and sediment deposition behind models of submerged vegetation with and without flexible leaves}

Zhenghong Hu ${ }^{1,2}$, Jiarui Lei ${ }^{2}$, Chao Liu ${ }^{1,2^{*}}$, Heidi Nepf ${ }^{2}$

${ }^{1}$ State Key Laboratory of Hydraulics and Mountain River Engineering, Sichuan University, Chengdu 610065, China.

${ }^{2}$ Department of Civil and Environmental Engineering, Massachusetts Institute of Technology, 77

Massachusetts Ave., Cambridge, MA 02139.

*Corresponding author: liuchaoscu@vip.qq.com 


\begin{abstract}
This laboratory study explored the flow structure and pattern of the deposition directly downstream of submerged patches of vegetation, focusing on whether, or not, the presence of flexible trailing leaves enhanced deposition. Both leaves of different length and patches of different geometry (circular and channel-spanning) were considered. The study defined the length of wake within which the velocity was diminished and net deposition was enhanced. The model sediment represented an organic or mineral solid smaller than fine sand. For a channel-spanning, submerged patch, recirculation or turbulent diffusion in the $x-z$ (streamwise-vertical) plane set the wake length within which velocity was diminished and deposition was enhanced. This length was greater for patches with lower stem densities, because greater flow through the patch displaced the recirculating eddy farther downstream. In addition, for a channel-spanning patch, the presence of flexible trailing leaves extended the wake length, which in turn increased the length of the deposition region. In contrast, for a circular patch, the wake contained oscillations in the $x-y$ (streamwise - lateral) plane. The onset of the wake unsteadiness set the length of the deposition region. Because the presence of flexible trailing leaves on a circular patch did not affect the formation distance for the wake oscillation, the length of the deposition region was unchanged with the addition of the flexible trailing leaves. For both circular and channel-spanning patches, a longer deposition region was associated with a larger deposition mass.
\end{abstract}

Key words: aquatic vegetation, wake structure, sediment deposition, ecohydraulics. 


\section{Introduction}

Aquatic vegetation plays an important role in shallow river ecosystems. It provides habitat for aquatic animals (Kemp et al. 2000). It also improves water quality by taking up nutrients and trapping heavy metals associated with suspended sediment (Brookshire and Dwire 2003, Cotton et al. 2006). The adjustment of flow around finite patches of vegetation influences the spatial distribution of sediment deposition and erosion (Rominger et al. 2010; Gurnell et al., 2012, Jones et al. 2012). For example, net deposition is enhanced in the wake downstream of a finite patch; erosion is observed at the lateral edge of a patch; and within the patch, both deposition and erosion are observed (Bouma et al., 2007; Tanaka and Yagisawa, 2010; Schulz et al. 2003; Follett et al. 2012; Shi et al. 2016, Liu and Nepf 2016). By producing a nutrient-rich substrate, regions of enhanced fine-particle deposition around patches of vegetation can drive patterns of future growth (Kondziolka and Nepf, 2014; Gurnell, 2014; Lima et al. 2015). From lab experiments, we have a good understanding of how the geometry of a rigid patch of emergent (Chen et al. 2012) or submerged (Liu et al. 2018) model vegetation impacts sediment deposition in the wake of a patch. However, most submerged vegetation is not rigid, and many submerged plants have flexible branches and leaves that bend with the current, producing a length of trailing leaves that overhang the bed. This study examined whether, or not, the presence of trailing leaves enhance deposition downstream of patches. Both leaves of different length and patches of different geometry (circular and channel-spanning) were considered.

Several researchers have investigated the flow and deposition within and around finite patches of emergent vegetation, i.e., vegetation that occupies the full water depth (Tanaka and Yagisawa, 2010; Zong and Nepf, 2011; Chen et al., 2012; Liu and Nepf, 2016; Shi et al., 2016). The vegetation was modeled with rigid circular cylinders placed in staggered or random arrangements within a circular patch. This is a reasonable mimic for rigid, emergent vegetation, such as reeds, sedges, and mangroves. Because the patch extends over the flow depth, the flow adjustment is predominantly two-dimensional in the horizontal $(x-y)$ plane, with some flow deflected laterally around the patch and some flow passing through the patch. Because some flow passes through the patch into the wake (bleed flow), the wake is different from that behind a solid body (Zong and Nepf, 2011; Nicolle and Eames, 2011; Chang and Constantinescu, 2015). Specifically, the von Karman vortex street does not occur directly behind the porous patch, as it would occur for a solid body. Instead, it appears at a distance $L_{x y}$ downstream from the trailing edge of the patch, which will be referred to as the formation distance (see Figure 1a, Zong and Nepf, 2011; Nicolle and Eames, 2011; 
Chang and Constantinescu, 2015). The distance $L_{x y}$ increases with decreasing stem density, $n$, and decreasing solid volume fraction, $\phi\left(=\pi n d^{2} / 4\right.$, with stem diameter $\left.d\right)$, until the bleed flow becomes strong enough to stabilize the shear layers, at which point no von Karman vortices are produced, but an unsteady wake flapping may still be present (Castro, 1971). Several studies have shown that the vortices are suppressed when $\phi<0.04$ to 0.05 (Nicolle and Eames 2011; Chang and Constantinescu 2015; Zong and Nepf 2011). Between the patch trailing edge and the formation distance $L_{x y}$ the wake exhibits reduced velocity and reduced turbulence intensity, which leads to enhanced deposition, relative to the free stream (e.g. Chen et al. 2012, Shi et al. 2016). By enhancing the deposition of nutrients and fine sediment, the region defined by $L_{x y}$ provides favorable conditions for the future growth and expansion of the vegetated region (Gurnell et al. 2012, Jones et al. 2012).

Other previous studies have described the flow near channel-spanning patches of submerged vegetation, i.e., vegetation that occupies the full channel width (Folkard, 2005, 2011; Chen et al. 2013). In these cases, the flow is deflected over the submerged vegetation, forming a shear layer in the vertical $x$ - $z$ plane, and the wake contains a recirculation eddy (Folkard, 2005). The distance $L_{x z}$ separates the trailing edge of the patch and the leading edge of the $x-z$ plane recirculating eddy (Figure 1b). As with the emergent patches discussed in the previous paragraph, $L_{x z}$ is expected to be a function of stem density. This relationship has been observed for two-dimensional fences (Perera, 1981). Specifically, for solid fences the recirculating eddy is observed directly downstream $\left(L_{x z}=0\right)$. However, for porous fences, bleed flow into wake shifts the recirculating eddy downstream, such that $L_{x z}$ increases with increasing fence porosity, which is analogous to decreasing $\phi$.

In nature, vegetation patches may be both submerged and of finite width, creating wakes that have shear layers in both the horizontal and vertical directions, with the potential to form vortices and recirculating eddies in both the $x-y$ and $x-z$ planes. This was confirmed using vegetation models constructed from circular arrays of rigid cylinders (Taddei et al. 2016, Liu et al. 2018). Taddei et al. (2016) confirmed the downstream shift of the recirculating eddy with decreasing solid volume fraction, and the absence of recirculation for $\phi<0.09$. Liu et al. (2018) reported the influence of patch height $(h)$ to width $(B)$ aspect ratio. Specifically, a vertical recirculation was observed behind wide patches $(h<B)$, whereas a von Karman vortex street was observed behind narrow patches $(h>B)$. Further, the minimum patch dimension ( $h$ or $B$ ) determined the length of quiescent wake within which deposition was enhanced.

This paper builds on the studies described above by considering the impact of more complex plant morphology 
on both the flow structure and sediment deposition within the wake. In contrast to the previous studies, which only considered rigid plant mimics, this study considered the impact of flexible leaves. The first group of experiments considered how the presence of flexible leaves of different length changed the wake structure and deposition produced by channel-spanning patches. The second group of experiments considered the impact of flexible leaves on circular patch wakes.

\section{Experimental Methods}

Experiments were carried out in a 3.75 -m long and $0.4-\mathrm{m}$ wide water channel with water depth $H=14.0 \pm 0.1 \mathrm{~cm}$. The flume bed was horizontal. The channel average velocity $\left(U_{0}\right)$ was $9.1 \mathrm{~cm} \mathrm{~s}^{-1}$. The flow was turbulent in all cases, with Reynolds number $\operatorname{Re}\left(=U_{0} R / v\right)=7,494$, in which $R$ is the hydraulic radius and $v\left(=0.01 \mathrm{~cm}^{2} / \mathrm{s}\right)$ is the kinematic viscosity. Model patches were constructed from rigid dowels of diameter $d=0.36 \mathrm{~cm}$ and leaves cut from low-density polyethylene (LDEP). The stem Reynolds number, $\operatorname{Re}_{d}(=U d / v)=250$ to 364 was estimated using the velocity at the leading edge of the patch $(U)$ and was consistently above the threshold $R e_{d}=120$, indicating that stem-scale turbulence was present over some extent inside the patch (Liu and Nepf 2016).

The model plants were inserted into a staggered arrangement of holes in a PVC baseboard. Group A included submerged, channel-spanning patches constructed from rigid dowels and from dowels with flexible leaves (Table 1). Rigid dowels of height $h_{\text {stem }}=7 \mathrm{~cm}$ were used to construct patches extending $10 \mathrm{~cm}$ in the streamwise direction at stem density $n=2.03$ stems $\mathrm{cm}^{-2}$ and $1.43 \mathrm{stems} \mathrm{cm}^{-2}$, corresponding to frontal area per patch volume $a(=n d)=0.73$

$\mathrm{cm}^{-1}$ and $0.51 \mathrm{~cm}^{-1}$, and solid volume fraction $\phi=0.21$ and 0.15 , respectively. Channel-spanning patches with flexible leaves were constructed from 2-cm high dowels with stem density $n=1.43 \mathrm{~cm}^{-2}$. Three LDPE leaves were attached to each dowel. Each leaf was $0.05 \mathrm{~mm}$ thick and $3 \mathrm{~mm}$ wide. Three leaf lengths were considered: $L_{\text {leaf }}=6,12$ and 26 $\mathrm{cm}$. In flow, these produced a canopy height $\left(h_{\text {canopy }}\right)$ between 6.5 and $7 \mathrm{~cm}$. Experiments in Group B included circular patches with width $B=10 \mathrm{~cm}$ positioned at the center of the channel (Figure $2 \mathrm{a}$ ). The patch Reynolds number was $R e_{B}$ $\left(=U_{0} B / v\right)=9,100$ to 36,400 . Submerged circular patches were constructed from 2-cm high dowels $\left(h_{\text {stem }}=2 \mathrm{~cm}\right)$, each with 3 LDPE leaves (Figure 2b). The leaf lengths in Group B ranged from $L_{\text {leaf }}=6$ to $33 \mathrm{~cm}$, producing canopy heights of 6.5 to $8 \mathrm{~cm}$. For all experiments, the submerged patches were designed to achieve a submergence ratio close to 0.5 for both rigid $\left(h_{\text {stem }} / H\right)$ and flexible $\left(h_{\text {canopy }} / H\right)$ patches, which is comparable to field condition reported in 
Sand-Jensen and Pedersen (2008).

The coordinate system was chosen such that $x$ was in the flow direction, and $x=0$ was located at the trailing edge of the stem region. The lateral coordinate was $y$, with $y=0$ located at the centerline of the channel and the patches. The vertical coordinate was $z$, with $z=0$ at the bed. A Nortek Vectrino (acoustic Doppler velocimeter, ADV) measured the velocity $(u, v, w)$ in coordinate directions $(x, y, z)$, respectively. At each measurement point the ADV recorded velocity at $25 \mathrm{~Hz}$ for $240 \mathrm{~s}$. The velocity data was de-spiked following the method of Goring and Nikora (2002). The instantaneous velocity was decomposed into its time average $(\bar{u}, \bar{v}$ and $\bar{w})$ and fluctuating $\left(u^{\prime}, v^{\prime}\right.$ and $\left.w^{\prime}\right)$ components. The turbulent kinetic energy was defined as $T K E=0.5\left(\overline{u^{\prime 2}}+\overline{v^{\prime 2}}+\overline{w^{\prime 2}}\right)$. The lateral velocity spectrum $S_{v v}$ was obtained using Welch's method found in the MATLAB Signal Processing Toolbox using a window size of 400 and an overlap of $8 \%$. The spectra were used to determine whether the wake oscillations were sufficiently coherent to stand out against the channel turbulence. The presence of von Karman vortices in the wakes of porous patches with $\phi>0.05$ has been demonstrated in previous studies (Nicolle and Eames, 2011; Zong and Nepf, 2011; Chang and Constantinescu, 2015). Specifically, downstream of a porous circular patch with $\phi>0.05$, a vortex street generates a peak in the $S_{v v}$ spectrum at frequency $f$ consistent with the Strouhal number $S t=f D / U_{0} \approx 0.14$ to 0.24 , as described in detail in Zong and Nepf(2011).

Flow visualization with fluorescein dye was used to reveal the flow structure in the patch wake. The fluorescein was excited using an ultraviolet lamp. For the channel-spanning patches (Group A), the tracer was injected at the top and center of the model vegetation, the UV light was placed above the flume, and the camera viewed the flume through the sidewall. For circular patches (Group B), additional images were collected with a camera above the flume and tracer injected at the outer-most edges of the patch. In these cases the UV light source was placed on the side of the flume. White tape on the flume bed marked $10-\mathrm{cm}$ intervals along the centerline starting at $x=0$. Images were recorded for $30 \mathrm{~s}$ at 20 frames per second. Using the Matlab image processing toolbox, 600 pictures were extracted from the video and used to construct a time-average image (e.g. Figure 3). A longitudinal transect of pixel intensity at $z=1 / 2 h_{\text {canopy }}$ was extracted from the time-averaged image (e.g. Figure 3). The pixel intensity transect was used to infer the length-scales of wake evolution $\left(L_{x y}\right.$ and $\left.L_{x z}\right)$ discussed in the Introduction. For example, measuring from the trailing edge of the stem region $(x=0)$, the position at which the pixel intensity increased above the background (pixel value 50) was defined as $L_{l}$, denoting the region never exposed to tracer. Beyond $L_{l}$ the pixel intensity rose quickly. 
$L_{2}$ was chosen at the point where the variation in pixel intensity began to level off (see Figure 3). We note that the pixel intensity represented an integration of dye concentration across the channel width, but since the dye was injected at the centerline, it was a fair representation of the tracer trajectory at the centerline. In a similar configuration with a rigid patch, Liu et al. (2018) noted the presence of a recirculating eddy in the $x$-z plane and documented the migration of its leading edge between two points in the channel, which corresponded to $L_{1}$ and $L_{2}$ in the pixel intensity transect (please see Figure 2 in Liu et al. 2018). However, a recirculating eddy was not present in all cases (e.g. case A2), and when the eddy was absent, the dye moved downward and reached the bed through the action of vertical turbulent diffusion. Therefore, $x=L_{x z}$ indicated the position where dye reached the channel bed either by recirculation or smaller-scale mixing. The distance $L_{x z}$ was estimated as $L_{x z}=\left(L_{1}+L_{2}\right) / 2$ with uncertainty $\left(L_{2}-L_{1}\right) / 2 . L_{x y}$ was defined in a similar way using images in the plan view.

Deposition experiments used model sediment chosen to represent organic matter carried in suspension in natural rivers, because it is the retention of organic matter that provides nutrients needed for vegetation growth. That is, we are interested in sediment that is normally carried in suspension, but which may deposit in the patch wakes where velocity and turbulence intensity are reduced (Chen et al. 2012, Shi et al. 2016). Sediment is carried predominantly in suspension when the ratio of settling velocity $\left(w_{s}\right)$ to bed friction velocity $(u *)$ is below $w_{s} / u_{*}=0.5$ (e.g. Chap 10 , Julien, 1995). The current experiment used glass spheres (Potters Industries, Valley Forge, PA) with density $\rho_{p}=2.5$ $\mathrm{g} / \mathrm{cm}^{3}$ and median diameter $d_{50}=7.1 \mu \mathrm{m}$, for which $w_{s} / u_{*}=0.005$. These particles do not represent a specific type of sediment; instead, they could represent any silt, fine sand or organic floc that is carried in suspension.

The net deposition was measured using glass microscope slides, which are inexpensive and easily cut to an appropriate size $(2.5 \mathrm{~cm} \times 2.5 \mathrm{~cm})$. The slides were initially weighed and then placed at predetermined locations on the channel bed (Figure 4) under no-flow conditions. The flow depth was slowly increased to the target depth $(14 \mathrm{~cm})$, and the pump speed was then slowly increased to the target channel-average velocity $(9.1 \mathrm{~cm} / \mathrm{s})$. To start a deposition experiment, $93.4 \mathrm{~g}$ of the particles were mixed thoroughly in a large plastic container and slowly added to the tail box. The pump circulated both the water and the particles to the head box, from which they re-entered the test section. The particles were mixed through the action of the pump. Because the particle slurry was added uniformly over one circulation period, the particles were mixed to a uniform initial concentration $\left(105 \mathrm{gm}^{-3}\right)$ within 2 minutes. The experiment was run for four hours, much longer than the initial mixing period. Because a fixed volume of water and 
sediment was recirculated through the channel, as sediment deposited, the concentration in the water declined, i.e. the sediment transport was not steady. After four hours, the pump speed was reduced slowly to avoid the generation of waves, and the channel drained in 20 minutes. Liu and Nepf (2016) reported that the net deposition during a 20-min draining period was spatially uniform and accounted for $9 \%$ of the total deposition that occurred during an experiment lasting 4 hours and 20 minutes in total; thus, the net deposition during the draining period was insignificant and did not alter the spatial distribution of deposits formed during the 4-hour experiment. Three heat lamps placed along the flume accelerated the drying of the slides. After 12 hours under heat lamps, most of the moisture had dissipated, and the deposits adhered to the slides. The slides were then gently moved to a $50{ }^{\circ} \mathrm{C}$ oven for another four hours to remove the remaining moisture. The slides were reweighed, and the difference in the weight before and after the experiment was taken to represent the net deposition. Each condition was run twice and the replicate standard deviation (1/2 the difference between replicates) was used as an indication of agreement between replicates.

To describe the deposition pattern in the wakes, a deposition length scale, $L_{D e p}$, was defined in the following manner. The deposition in the open channel, $\operatorname{Dep}_{0}\left[\mathrm{mg}\right.$ per $\left.\mathrm{cm}^{2}\right]$ was estimated as the average net deposition on the three slides positioned farthest upstream $(x / D=-3,-2.7,-2.3)$. In most cases, deposition in the patch wake was higher than $\mathrm{Dep}_{0}$ over several slides. For example, Figure 5 depicts the deposition measured for the two replicates of Case A2, for which net deposition was elevated above $D e p_{0}$ in the wake. $L_{D e p}$ was defined as the distance from the trailing edge of the stem region to the point at which the deposition declined to a value at or below $D e p_{0}$. An estimate of $L_{D e p}$ was made for each replicate. The mean and replicate standard deviation was reported in Table 1 . For Case A2, $L_{D e p}=$ $40 \pm 3 \mathrm{~cm}$. Note that the deposition was not elevated at the slide directly adjacent to the stem region $(x / D=0+$ in Figure 5). This was due to the presence of stem-generated turbulence that inhibits deposition inside and directly downstream of the patch (Chen et al. 2012, Liu and Nepf, 2016). Finally, the total mass deposited per wake width $\left(M_{w}\right)$

was estimated from the spatial-average deposition over length $L_{D e p}$, denoted as $D e p_{w}\left[\mathrm{mg} \mathrm{cm}{ }^{-2}\right]$. Specifically, $M_{w}=$ $L_{\text {Dep }} \operatorname{Dep}_{w}$ (Table 1).

\section{Results and Discussion}

\subsection{Rigid, submerged, channel spanning patch}

Previous studies of emergent circular patches, for which the dominant wake flow structure is a von Karman vortex 
street in the $x-y$ plane, have shown that the deposition length, $L_{D e p}$, is set by the vortex formation distance, $L_{x y}$, which is sensitive to the stem density. As stem density decreases, allowing more flow to pass through the patch, the formation distance is pushed farther downstream, and $L_{D e p}$ increases in step with $L_{x y}$ (Zong and Nepf 2011, Chen et al. 2012). This study considered whether a similar dependence between $L_{x z}, L_{D e p}$, and the solid volume fraction $(\phi)$ existed for channel-spanning submerged patches, for which a recirculation eddy may occur in the $x-z$ plane. The velocity fields around two channel-spanning patches of solid volume fraction 0.21 (case A1) and 0.15 (case A2) are shown in Figure 6. The velocity field was confirmed to be two-dimensional by comparing the depth-averaged velocity at each longitudinal position upstream and along the patch. Specifically, the depth-average velocity calculated from the velocity profiles measured at $x / D=-3$ (upstream), 2, 4 and 6 , and found to differ by less than $5 \%$. For the higher solid volume fraction (Figure 6a), the time-averaged velocity directly behind the patch was diminished to nearly zero $(\approx 0.5 \mathrm{~cm} / \mathrm{s})$, and a time-mean recirculating region was present between $x / D=0.5$ and $4(D=10 \mathrm{~cm}$, Figure $6 \mathrm{a})$. The flow visualization established the distance $L_{x z}=20 \pm 3 \mathrm{~cm}$ (Figure 7f). In contrast, for the lower solid volume fraction, $(\phi=0.15$, case A2, Figure $6 \mathrm{~b})$, the velocity leaving the patch was higher $(3.6 \mathrm{~cm} / \mathrm{s})$, resulting in weaker shear in the vertical (x-z) plane. In this case the velocity vectors did not indicate recirculation (Figure 6b), suggesting that the flow entering the wake was strong enough to inhibit the formation of the recirculation in the $x-z$ plane. That is, the $x-z$ plane recirculation was observed behind the higher solid volume fraction patch, but not behind the lower solid volume fraction patch, consistent with observations behind porous fences (Perera, 1981). Without the vertical mixing provided by a recirculating eddy, the vertical diffusion of dye towards the bed occurred more gradually, producing a longer $L_{x z}=42 \pm 4 \mathrm{~cm}$ (Figure $7 \mathrm{~g}$ ). In this case $L_{x z}$ was defined by vertical diffusion. Finally, the region of elevated deposition $\left(L_{D e p}\right)$ in the A1 and A2 wakes (Figure 7a and 7b, respectively) correlated with the wake length-scale $\left(L_{x z}\right)$ revealed in the flow visualization (Figure $7 \mathrm{f}$ and $7 \mathrm{~g}$ ) regardless of the relative influence of a recirculating eddy or vertical turbulent diffusion, with a longer region of deposition associated with the lower stem density (case A2). Specifically, in case A1, the enhanced deposition region, $L_{D e p}=22 \pm 1 \mathrm{~cm}$, was the same as $L_{x z}=20 \pm 3 \mathrm{~cm}$, considering the difference between replicates. Similarly, in case A2, $L_{D e p}=40 \pm 3 \mathrm{~cm}$ and $L_{x z}=42 \pm 4 \mathrm{~cm}$. These observations suggested that the region of enhanced deposition in the wake of a wide patch was set by distance, $L_{x z}$, and that both $L_{x z}$ and $L_{D e p}$ increased with decreasing solid volume fraction $(\phi)$. 


\subsection{Submerged, channel-spanning patch with leaves}

Many species of submerged vegetation have long, flexible leaves. Cases A3, A4 and A5 explored the impact of leaf length on deposition, using $L_{\text {leaf }}=6,12$, and $26 \mathrm{~cm}$. The flexible leaves decrease the velocity behind the patch, compared to the velocity behind a rigid patch with the same stem density (case A2, Figure 6b). For example, the velocity downstream from the longest leaves was less than $0.5 \mathrm{~cm} / \mathrm{s}$ (case A5 with the leave length of $26 \mathrm{~cm}$, Figure $6 \mathrm{c})$, which was smaller than the velocity $(3.6 \mathrm{~cm} / \mathrm{s})$ behind the rigid patch without leaves. The streamwise extent of the pronated leaves is shown with a dashed black line in Figure 7c, 7d, and 7e. Deposition was observed beneath the leaves and over some distance beyond the end of the leaves, so that as the leave length increased, the length of the deposition region also increased (Table 1). Specifically, deposition extended 10 to $15 \mathrm{~cm}$ beyond the end of the leaves. Flow visualization illustrated that the quiescent wake zone (denoted by the absence of tracer) also extended 10 to 15 $\mathrm{cm}$ beyond the trailing edge of the leaves (Figure $7 \mathrm{~h}, 7 \mathrm{i}, 7 \mathrm{j}$ ), which corresponded to $(1.6 \pm 0.4) h_{\text {canopy }}$, so that $L_{x z} \approx$ $L_{\text {leaf }}+(1.6 \pm 0.4) h_{\text {canopy }}$. By shifting the position of $L_{x z}$ downstream, the leaves created a longer region favorable to deposition, with $L_{D e p}=L_{\text {leaf }}+(1.6 \pm 0.7) h_{\text {canopy }}$ (data from cases A3, A4 and A5). As with the rigid canopies (Figure $7 \mathrm{a}$ and $7 \mathrm{~b}$ ), the length of the deposition region, $L_{D e p}$, closely tracked the wake length-scale, $L_{x z}$ (Table 1). Specifically, considering all channel spanning cases (A series, Table 1), $L_{D e p} / L_{x z}=1.04 \pm 0.07$ (SD).

The specific enhancement in net deposition $D e p_{w} / D e p_{0}$ was the same for the three leaf lengths (cases A3, A4 and A5). Specifically, $\operatorname{Dep}_{w} / \operatorname{Dep}_{0}=1.9 \pm 0.3$ (SD). However, since the length of deposition region $\left(L_{D e p}\right)$ increased with leaf length, the total mass deposited per wake width, $M_{w}\left(=D e p_{w} \times L_{D e p}\right)$ also increased with leaf length. Specifically, the largest total mass $\left(M_{w}=103 \pm 7 \mathrm{~g} \mathrm{~cm}^{-1}\right)$ occurred behind the patch with the longest leaves $L_{\text {leaf }}=26 \mathrm{~cm}$, compared to just $63 \pm 3 \mathrm{~g} \mathrm{~cm}^{-1}$ for $L_{\text {leaf }}=6 \mathrm{~cm}$ (Table 1$)$.

The presence of the leaves also influenced the turbulent kinetic energy (TKE) in the patch wake. Specifically, as the leaf length increased, the magnitude of TKE in the wake decreased (Figure 8). Previous studies have documented the motion (reconfiguration) of flexible plants in response to the passage of turbulent eddies (e.g. Plew et al. 2008, Huang et al. 2011, Rominger and Nepf 2014). The passive motion of the leaves weakens the turbulence (Ghisalberti and Nepf 2006). Siniscalchi and Nikora (2012) also note that the wave-like motions of the freshwater plant Glyceria fluitans reduced the turbulence in its wake. In this study, the patch with shorter leaves $\left(L_{\text {leaf }}=6 \mathrm{~cm}\right)$ exhibited less leaf motion and higher levels of TKE in the wake (Figure 8a). Conversely, the patches with longer leaves $\left(L_{\text {leaf }}=12\right.$ and 26 
$\mathrm{cm}$ ) exhibited greater motion and correspondingly lower turbulence in the wake (Figure $8 \mathrm{~b}$ and $8 \mathrm{c}$ ). Further, the extent of the patch wake in which turbulence was significantly reduced (dark blue dots) was consistent with the region of enhanced deposition, and this distance increased with increasing leaf length and increasing leaf motion. That is, our measurements suggested that the motion of flexible leaves damped turbulence (Figure 8) and enhanced deposition downstream the patches (Figure 7). It is interesting to note that Sand-Jensen (1998) also observed the movement of a trailing canopy of Ranunculus peltatus, but associated the movement of the trailing leaves with the erosion of the sediment downstream the patches. Given that the passive movement of flexible leaves has been shown to reduce turbulence (e.g. Siniscalchi and Nikora 2012), this association is difficult to explain.

\subsection{Submerged, circular patch with flexible leaves}

Cases B1 to B5 explored the impact of leaf length on the wake structure and deposition behind a circular patch, using leaves that extended 6 to $33 \mathrm{~cm}$ beyond the trailing edge of the root region (Figure 9). In these cases, the deposition length had little dependence on the leaf length. Specifically, across all leaf lengths, the deposition length was the same, $L_{D e p}=14 \pm 3 \mathrm{~cm}$ (Table 1), which was slightly larger than the diameter of the stem region $(D=10 \mathrm{~cm})$, i.e. $L_{D e p} / D=$ $1.4 \pm 0.3$. The lack of influence from leaf length can be further illustrated by plotting together the longitudinal profiles of net deposition for all five cases (B1 to B5, Figure 10). The pattern of deposition was nearly identical, despite the addition of leaves with length 6 to $33 \mathrm{~cm}$. Further, Liu et al. (2018) reported $L_{\text {Dep }}$ behind a rigid submerged patch with no leaves. Their circular patch had similar dimensions to this study, with diameter $D=10 \mathrm{~cm}$ and patch height $h_{\text {stem }}=$ $7 \mathrm{~cm}$ (see case A15 in Liu et al. 2018). For the patch without leaves Liu et al. reported $L_{D e p} / D=1.4 \pm 0.2$, which was the same as that observed here for patches with flexible leaves $(1.4 \pm 0.3)$, indicating that neither the addition of leaves or the length of the leaves impacted the deposition length-scale behind a circular patch. This result for submerged circular patches stands in strong contrast to the channel-spanning patch with leaves (Figure 7), for which $L_{\text {Dep }}$ increased with increasing leave length. Returning to the circular patches, for increasing leaf length $\left(L_{\text {leaf }}=12\right.$, 19 and $26 \mathrm{~cm}$, cases B2, B3, B4, respectively), the formation distance shifted from $L_{x y}=12 \pm 2$ to $19 \pm 2 \mathrm{~cm}$ (Table 1). For B1 and B5, the formation point was hidden beneath the leaves (Figure 9). For cases with measured $L_{x y}$, the deposition length was similar to the formation distance, with $L_{D e p} / L_{x y}=0.9 \pm 0.2$ (SD). These ratios indicated that for circular patches, the formation distance, $L_{x y}$, set the deposition length, $L_{D e p}$. 
Similar to the channel-spanning patch, the deposition enhancement in the circular patch wake $\left(\operatorname{Dep}_{w} / \operatorname{Dep}_{0}\right)$ was not impacted by the leaf length. Specifically, considering all circular patches with flexible leaves (cases B1-B5), $D e p_{w} / \operatorname{Dep}_{0}=1.7 \pm 0.2(\mathrm{SD})$, which was the same as that observed for channel-spanning patches with flexible leaves $\left(D e p_{w} / D e p_{0}=1.9 \pm 0.3\right)$, within uncertainty. Because both $L_{D e p}$ and $D e p_{w}$ were unaffected by leaf length in circular patches, the total deposition per wake width, $M_{w}=D e p_{w} L_{D e p}$, was also largely unaffected by leaf length. Specifically, $M_{w}$ was the same for $L_{\text {leaf }}=12,19,26$ and $33 \mathrm{~cm}$ (cases B2, B3, B4 and B5, respectively) within uncertainty (Table 1). However, a slightly smaller $M_{w}$ was observed for the patch with shortest leaves $L_{\text {leaf }}=6 \mathrm{~cm}$. This was consistent with the slightly shorter $L_{D e p}$ observed in this case (Table 1 ).

For the circular patches, the lateral instability at formation distance $L_{x y}$ occurred before any visual evidence of recirculation or mixing in the $x-z$ plane (i.e. $L_{x y}<L_{x z}$ ). The unsteadiness observed at distance $L_{x y}$ was not clearly a vortex street (Figure 11), and resembled more the flapping wake regime described in Castro (1971). Specifically, Castro (1971) noted that when bleed air through a porous plate was sufficient, it could prevent the shear layers from interacting, which inhibited the formation of the Karman vortex street. While no vortices form, the wake flaps at the same dominant frequency as observed for the Karman vortex street. Consistent with this description, for the submerged circular patches in this study, the wake oscillated back and forth at a frequency consistent with that expected for a von Karman vortex, here $0.1 \mathrm{~Hz}$ (Figure 11c). Specifically, the wake oscillated from one side (at time $t_{0}$; Figure 11a) to the other (at time $t_{0}+T_{k v} / 2$; Figure 11b), in which $T_{k v}=10 \mathrm{~s}$ is the period of the expected von Karman vortex. Further, the lateral velocity spectrum $S_{v v}$ had distinguishable peaks at $0.1 \mathrm{~Hz}$ (Figure 11c), which was consistent with the oscillation observed in the tracer (Figure 11a and b).

\section{Discussion}

The current study demonstrated that the recirculating eddies and instabilities formed in the wake of a submerged vegetation patch provided an important control on the length of the deposition region within the patch wake. For a channel-spanning patch, the formation of a recirculating eddy in the $x-z$ plane controlled the deposition length. The eddy-formation length was controlled by both the patch height and leaf length. For the circular patch, the formation of a lateral wake oscillation controlled the deposition length, and both the formation length and deposition length were controlled by the patch width. Given these distinct wake and deposition controls for wide versus narrow patches, it 
would be useful to understand the patch aspect ratio $(h / B)$ that defines the transition between the two conditions. Previous studies (Liu et al., 2018, Ortiz et al., 2013) were used to delineate the patch height to width aspect ratio at which the wake transitions between these two cases. In Liu et al. (2018) and Ortiz et al. (2013) the Reynolds number was $R e=7,494$ to 9,133 and 12,821 , respectively, in which $R e=U_{0} R / v$. When the patch was sufficiently submerged $(h / H<0.5)$, and had a low aspect ratio $(h / B<0.75)$, a von Karman vortex street was not observed (red symbols in Figure 12); otherwise, the von Karman vortex street was observed (blue symbols in Figure 12). This transition illustrated in the data shown in Figure 12 suggests that the minimum patch dimension ( $h$ vs $B$ ) controls the wake structure and deposition behavior. That is, the von Karman vortex forms at a distance set by patch width $B$ (Zong and Nepf, 2011), and the vertical plane recirculating eddy forms at a distance set by canopy height $h$ (Perera, 1981). Based on these scales, one would expect that if $h / B<\approx 1$, the vertical recirculation should form closer to the patch, and eliminate the $x-y$ plane vortex, which is consistent with the observed transition at $h / B \approx 0.75$.

Consistent with the lab observations, enhanced net deposition has been observed in the field in the wake of both emergent and submerged vegetation (Shulz et al. 2003; Bouma et al. 2005; Tanaka, and Yagisawa, 2010). Results from the present study can provide additional insight into field observations of wake deposition and its connection to growth patterns. For example, Callitriche cophocarpa is the dominant species in many northern European streams. This macrophyte grows flexible branches that form a submerged canopy extending some distance downstream from its stem zone, i.e. similar to the flexible leave mimics considered in this study. The patches of Callitriche cophocarpa grow predominantly in the downstream direction (Sand-Jensen and Madsen, 1992), consistent with the hypothesis that deposition in the wake downstream of the patch influences growth patterns by providing a nutrient rich substrate that promotes new growth (Gurnell et al, 2012, Jones et al. 2012). For this species, the canopy height $\left(h_{\text {canopy }}\right)$ to patch width $(D)$ ratio is $h_{\text {canopy }} / D=0.63 \pm 0.08$ (based on data in Table 1, Sand-Jensen and Pedersen, 2008), which suggests that the patch wakes are dominated by a vortex in the $x$-z plane (Figure 12). In fact, Sand-Jensen (1998) noted the presence of $x-z$ plane vortices in the wakes of Ranunculus peltatus (see point 2 in the summary of Sand-Jensen, 1998). Further, Sand-Jensen (1998) reported that flow acceleration downstream from Ranunculus peltatus patches was more pronounced for longer and wider patches, with stronger eddies forming at the rear. Because longer and wider patches would create a greater reduction in the velocity in the patch wake, the greater velocity difference from the free stream would produce stronger eddies in the patch wake. 
As a final note, the patterns of deposition observed in this paper corresponded to a single particle size. While the distribution of enhanced deposition (i.e., $L_{D e p}$ values) should apply across all sediment sizes, the magnitude of deposition enhancement within the wake will be function of sediment size. Shi et al. (2016) parameterized the particle size effect through the ratio of critical shear velocity for resuspension, $u_{* c}$, and the open-channel shear velocity, $u_{*}$. Specifically, the preferential deposition of material within a patch wake was only observed for $0.7<u_{*} / u_{* c}<3$, and the peak enhancement in wake deposition, relative to the adjacent bare-bed, occurred at $u_{*} / u_{* c}=1.6$. Outside this range, the deposition within the patch was not enhanced relative to the adjacent bare-bed, so that no positive feedback for growth could be generated. In addition, we note that the results of this study were obtained downstream of a dense patch of submerged vegetation (with $a h>0.1$ ); the large exit velocities that occur directly downstream from sparse patches (with $a h<0.1$ ) may alter the vortex structure and deposition patterns, and this behavior requires further investigation.

\section{Summary}

In the wake behind a patch of model submerged vegetation there is a strong correlation between the formation distance for wake recirculation and unsteadiness, $L_{x y}$ and $L_{x z}$, and the region of enhanced deposition, $L_{D e p}$, This correlation can provide ecologists with a framework for understanding the feedbacks between vegetation, flow, deposition and growth. For wide patches $(h / B<0.75)$, the wake is dominated by a recirculating eddy that forms in the $x-z$ plane, and $L_{x z} \sim h$. For wide patches, the recirculation eddy can be delayed by trailing leaves, so that the presence of leaves extends the deposition region, allowing for more rapid downstream growth. In addition, long leaves provide greater damping of turbulence within the wake. In contrast, for a narrow submerged patch, $h / B>$ 0.75 , at distance $L_{x y}$ from the patch the wake develops a von Karman vortex street or lateral wake oscillation in the $x-y$ plane, and $L_{x y}$ determines the length of the deposition region. Because the presence of flexible trailing leaves does not affect the formation of the von Karman vortex street or the onset of wake flapping, the length of the deposition region behind a narrow patch is unaffected by the length of flexible trailing leaves. The formation distance is sensitive to the stem density, with $L_{x z}$ and $L_{x y}$ increasing with decreasing solid volume fraction. Finally, the net deposition per bed area $\left(\mathrm{mg} \mathrm{cm}^{-2}\right)$ within the wake was the same for all wakes (channel-spanning 
and circular, and independent of leaf length). As a result of this, the total mass deposited within the wake (per wake width) increased with increasing $L_{D e p}$. Therefore, for wide patches $(h / B<0.75)$, the total mass deposited in the wake increases with leaf length, but for narrow patches $(h / B>0.75)$ the total mass deposited in the wake is unaffected by leaf length.

\section{Acknowledgements}

Zhenghong Hu and Chao Liu were finally supported by the National Natural Scientific Foundation of China (Grant Nos. 51609160 and 51539007). The funding for this project was provided by the US National Science Foundation (Grant No. EAR-1414499) and. Any opinions, findings, or recommendations expressed in this material are those of the author(s) and do not necessarily reflect the views of the National Science Foundation. 
Table 1 Experiment Parameters ${ }^{*}$

\begin{tabular}{|c|c|c|c|c|c|c|c|c|c|c|c|c|c|}
\hline Cases & $\begin{array}{r}\mathrm{S}= \\
\mathrm{E}= \\
\mathrm{FB}=\end{array}$ & $\begin{array}{l}\text { merged } \\
\text { ergent } \\
\text { igid } \\
\text { ble leaves }\end{array}$ & $\begin{array}{c}B \\
(\mathrm{~cm}) \\
\pm 0.2\end{array}$ & $\begin{array}{c}D \\
(\mathrm{~cm}) \\
\pm 0.2\end{array}$ & $\begin{array}{c}\phi \\
\pm 0.01\end{array}$ & $\begin{array}{l}h_{\text {stem }} \\
(\mathrm{cm}) \\
\pm 0.2\end{array}$ & $\begin{array}{l}h_{\text {canopy }} \\
(\mathrm{cm})\end{array}$ & $\begin{array}{l}L_{\text {leaf }} \\
(\mathrm{cm}) \\
\pm 0.5\end{array}$ & $L_{x z} / D$ & $L_{x y} / D$ & $L_{D e p} / D$ & $\operatorname{Dep}_{w} / \operatorname{Dep}_{0}$ & $M_{w}\left(\mathrm{~g} \mathrm{~cm}^{-1}\right)$ \\
\hline \multirow{4}{*}{$\begin{array}{c}\text { Channel } \\
\text { spanning } \\
\text { patches }\end{array}$} & A1 & $\mathrm{S}, \mathrm{R}$ & 40.0 & 10.0 & 0.21 & 7.0 & $7.0(0.2)$ & - & $2.0(0.3)$ & - & $2.2(0.1)$ & $1.8(0.0)$ & $60(3)$ \\
\hline & A3 & $\mathrm{S}, \mathrm{FB}$ & 40.0 & 10.0 & 0.15 & 2.0 & $6.5(0.5)$ & 6.0 & $1.9(0.3)$ & - & $2.1(0.1)$ & $2.1(0.4)$ & $63(3)$ \\
\hline & A4 & $\mathrm{S}, \mathrm{FB}$ & 40.0 & 10.0 & 0.15 & 2.0 & $7(1)$ & 12.0 & $2.2(0.4)$ & - & $2.4(0.1)$ & $2.1(0.1)$ & $73(6)$ \\
\hline & A5 & $\mathrm{S}, \mathrm{FB}$ & 40.0 & 10.0 & 0.15 & 2.0 & $7(1)$ & 26.0 & $3.2(0.4)$ & - & $3.2(0.2)$ & $1.5(0.2)$ & $103(7)$ \\
\hline \multirow{2}{*}{$\begin{array}{l}\text { Circular } \\
\text { patches }\end{array}$} & B1 & $\mathrm{S}, \mathrm{FB}$ & 10.0 & 10.0 & 0.15 & 2.0 & $6.5(0.5)$ & 6.0 & $1.2(0.2)$ & $\leq L_{\text {leaf }}$ & $1.1(0.2)$ & $1.9(0.4)$ & $29(6)$ \\
\hline & B5 & $\mathrm{S}, \mathrm{FB}$ & 10.0 & 10.0 & 0.15 & 2.0 & $8(1)$ & 33.0 & $4.0(0.2)$ & $\leq L_{b}$ & $1.4(0.3)$ & $1.7(0.1)$ & 47 (12) \\
\hline
\end{tabular}

${ }^{*} B$ is patch width and $D$ is patch length in the streamwise direction. In A1 to A5 a submerged patch spanned the channel width, so $B$ was the channel width $(=40 \mathrm{~cm})$. In B1 to B5 the patch was circular with diameter $D(=B)=10 \mathrm{~cm}$. In all cases the channel-average velocity was $U_{0}=9.1 \pm 0.1 \mathrm{~cm} / \mathrm{s}$, and the water depth was $H=14.0 \pm 0.1 \mathrm{~cm}$. The solid volume fraction $\phi=\pi n d^{2} / 4$, with $n$ the stem density within the patch and $d$ the stem diameter. Numbers in brackets are uncertainties. The uncertainty of $h_{\text {canopy }}$ is measurement uncertainty. The uncertainty in $L_{x z}, L_{x y}$ and $L_{D e p}$ are the replicate standard deviations. $D e p_{w}$ was the mean net deposition over $L_{D e p}$. Dep 0 was the mean net deposition on the three slides positioned farthest upstream $(x / D=-3,-2.7,-2.3) . M_{w}\left(=D e p_{w} \times L_{D e p}\right)$ was the total mass of deposition on the centerline. 


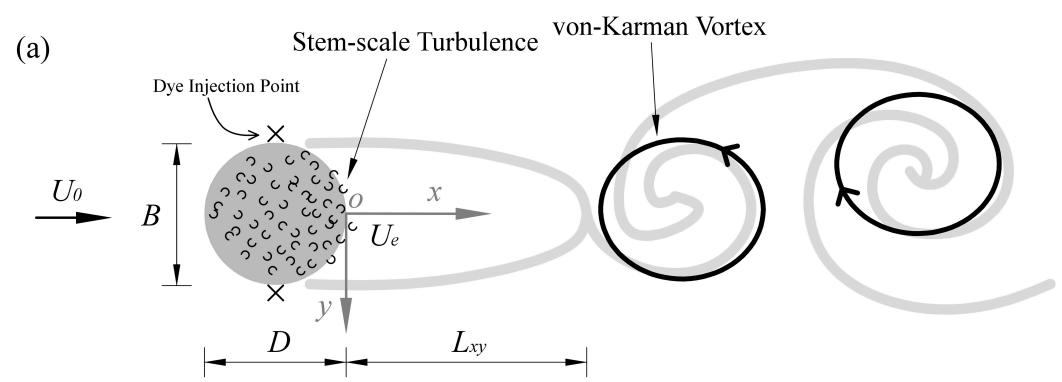

(b)

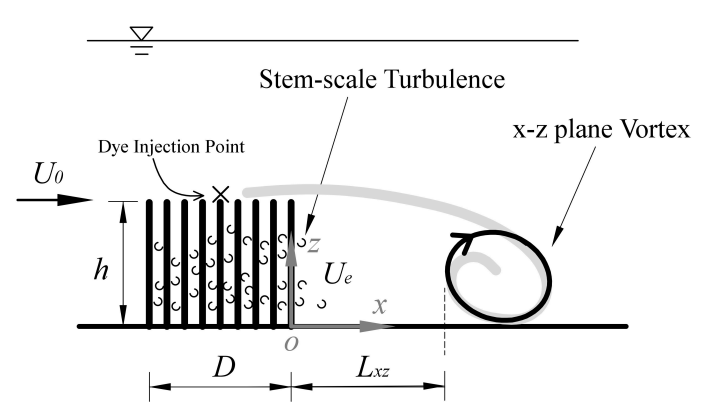

Figure 1. The schematic of flow structures behind a submerged patch consisting of rigid cylinders, (a) plan view; and (b) side view. $L_{x y}$ is the distance from the trailing edge of the patch to the onset of the von Karman vortex street. $L_{x z}$ is the distance from the trailing edge of the patch to the leading edge of recirculating eddy, which will be called the $x-z$ plane eddy. Patch height $(h)$, patch width $(B)$ and length (D) are denoted. This image is adapted from Liu et al. (2018). 
(a)

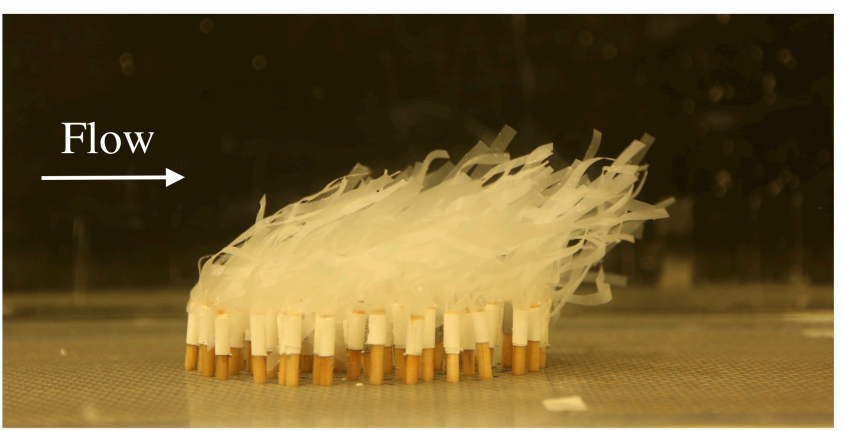

(b)

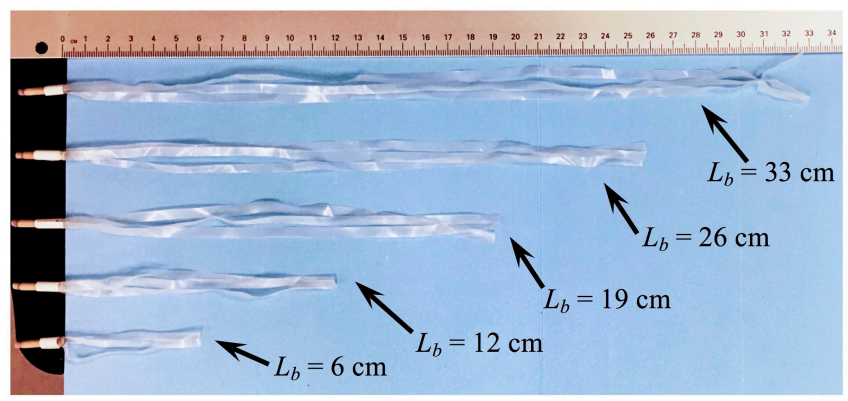

Figure 2. (a) A circular patch with flexible leaves of length $L_{\text {leaf }}=6 \mathrm{~cm}$; (b) Five leave lengths $L_{\text {leaf }}=6$, 12, 19, 26 and $33 \mathrm{~cm}$ (Table 1). Each stem had three flexible leaves, which were $0.05 \mathrm{~mm}$ thick and 3 mm wide. 


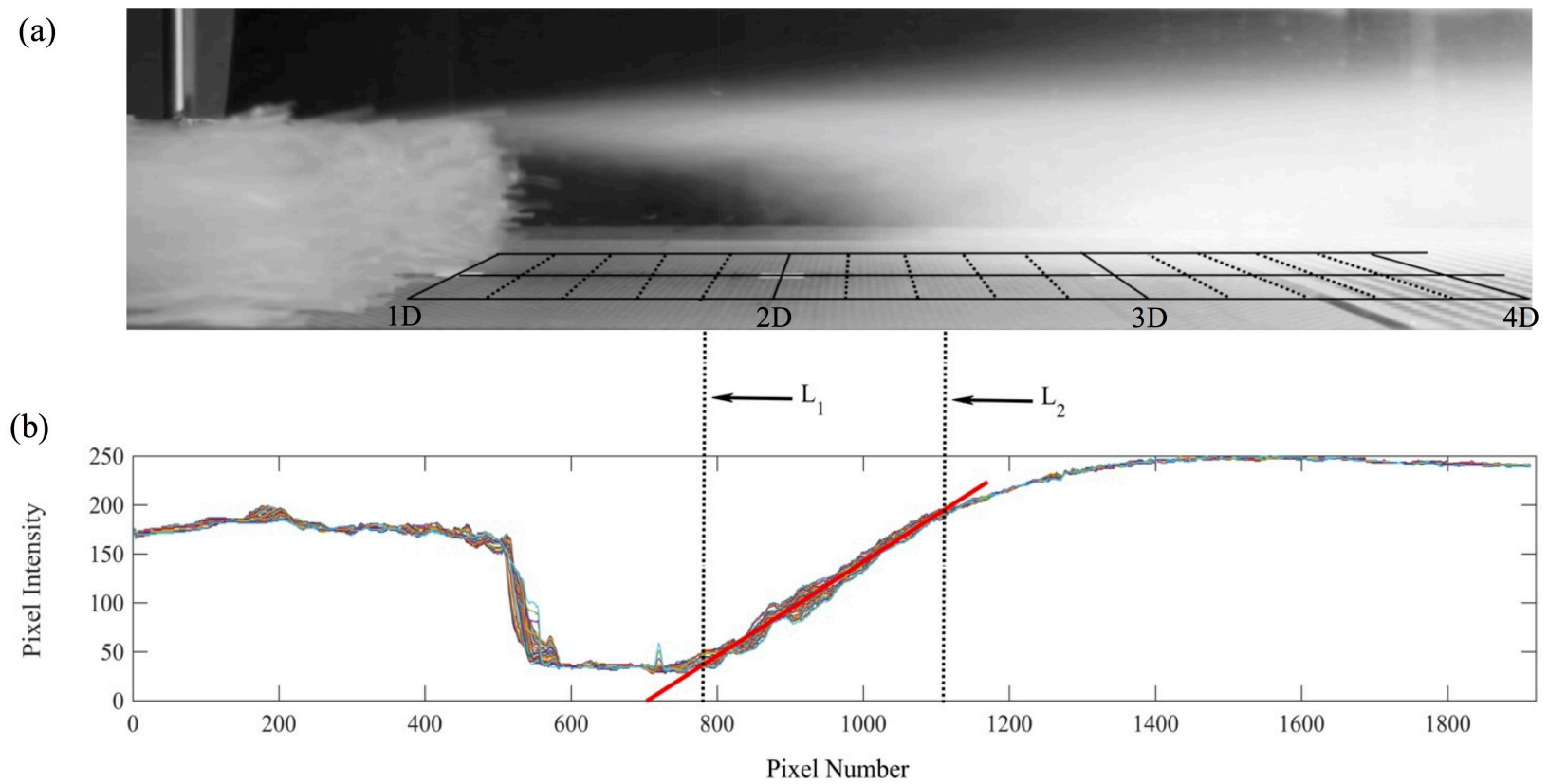

Figure 3. Example of image processing for case A4 with a channel-spanning patch with flexible leaves of length $L_{\text {leaf }}=12 \mathrm{~cm}$. Fluorescein dye was injected at the top center of the canopy. The end of the stem region was $x=0$, and positions $x=1 D, 2 D, 3 D$ and $4 D$ are marked white tags on the bed. (a) time-averaged image (b) longitudinal transect of time-averaged pixel intensity at $z=1 / 2 h_{\text {canopy }}$. Transect represents a vertical average over \pm 20 pixels $( \pm 0.5 \mathrm{~cm})$. The black grids account for parallax. $L_{l}$ was chosen at the position where the pixel intensity rose above 50, the black background level. Beyond $L_{l}$ pixel intensity rose quickly, with a slope indicated by the red line. $L_{2}$ was chosen at the point at which the variation in pixel intensity began to level off, i.e. moved off the slope of red line. 

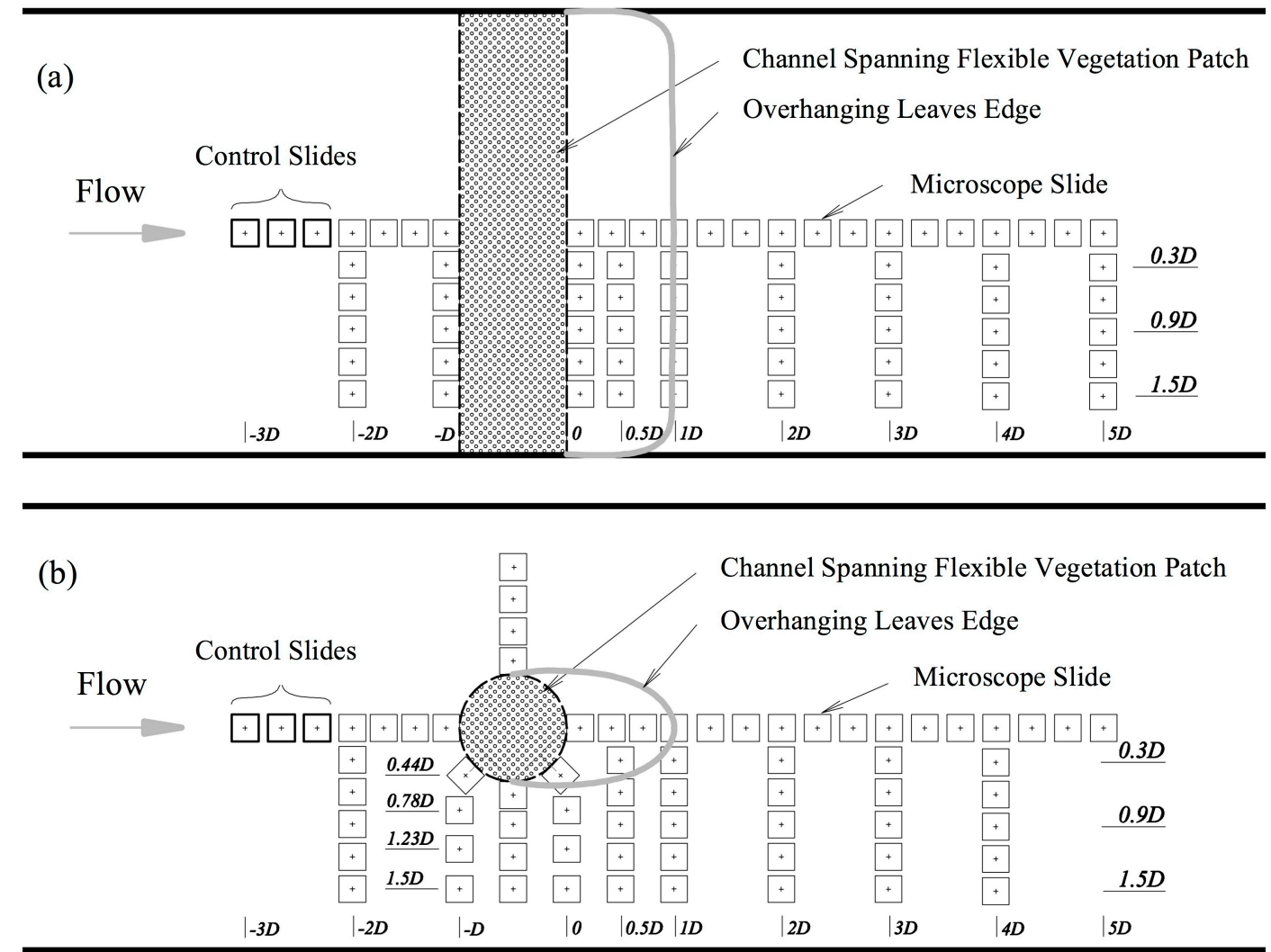

Figure 4. Top view of experimental setup, showing the position of each microscope slide (square with center dot). Hatched area indicates the stem region, consisting of rigid circular cylinders. When present, flexible leaves extended different lengths in the streamwise direction. The maximum leave length is shown with light grey curve. (a) channel-spanning patch with stem region extending $10 \mathrm{~cm}$ in streamwise direction (b) circular patch with stem regions of diameter $D=10 \mathrm{~cm}$. The three control slides positioned farthest upstream $(x / D=-3,-2.7$ and -2.3$)$ were used to estimate as the average net deposition in the open channel. 


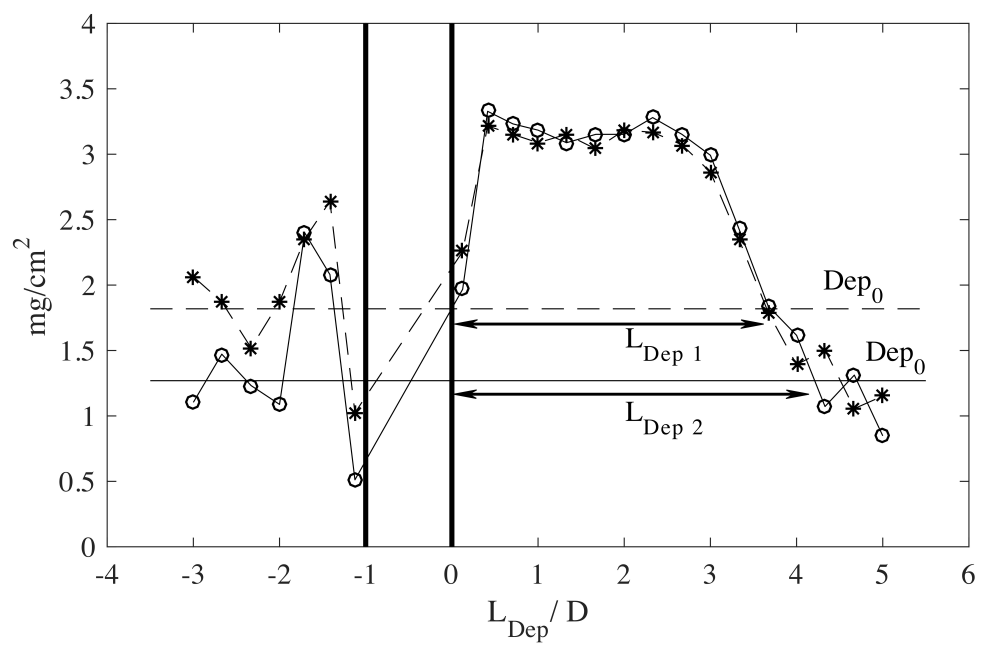

Figure 5. Net deposition measured in replicate 1 (circles) and replicate 2 (asterisks) for case A2. For each replicate the open channel deposition, $\operatorname{Dep}_{0}$, was estimated as the mean net deposition at the three slides located farthest upstream $(x / D=-3,-2.7,-2.3)$, and is shown with a dashed and a solid horizontal lines for replicate 1 and 2, respectively. The length of the enhanced deposition region, $L_{D e p}$, was determined from the points with deposition greater than $D e p_{0}$. For replicate 1 and $2, L_{D e p} / D=3.7$ and 4.2 , respectively, resulting in $L_{D e p} / D=4.0 \pm 0.3$. 

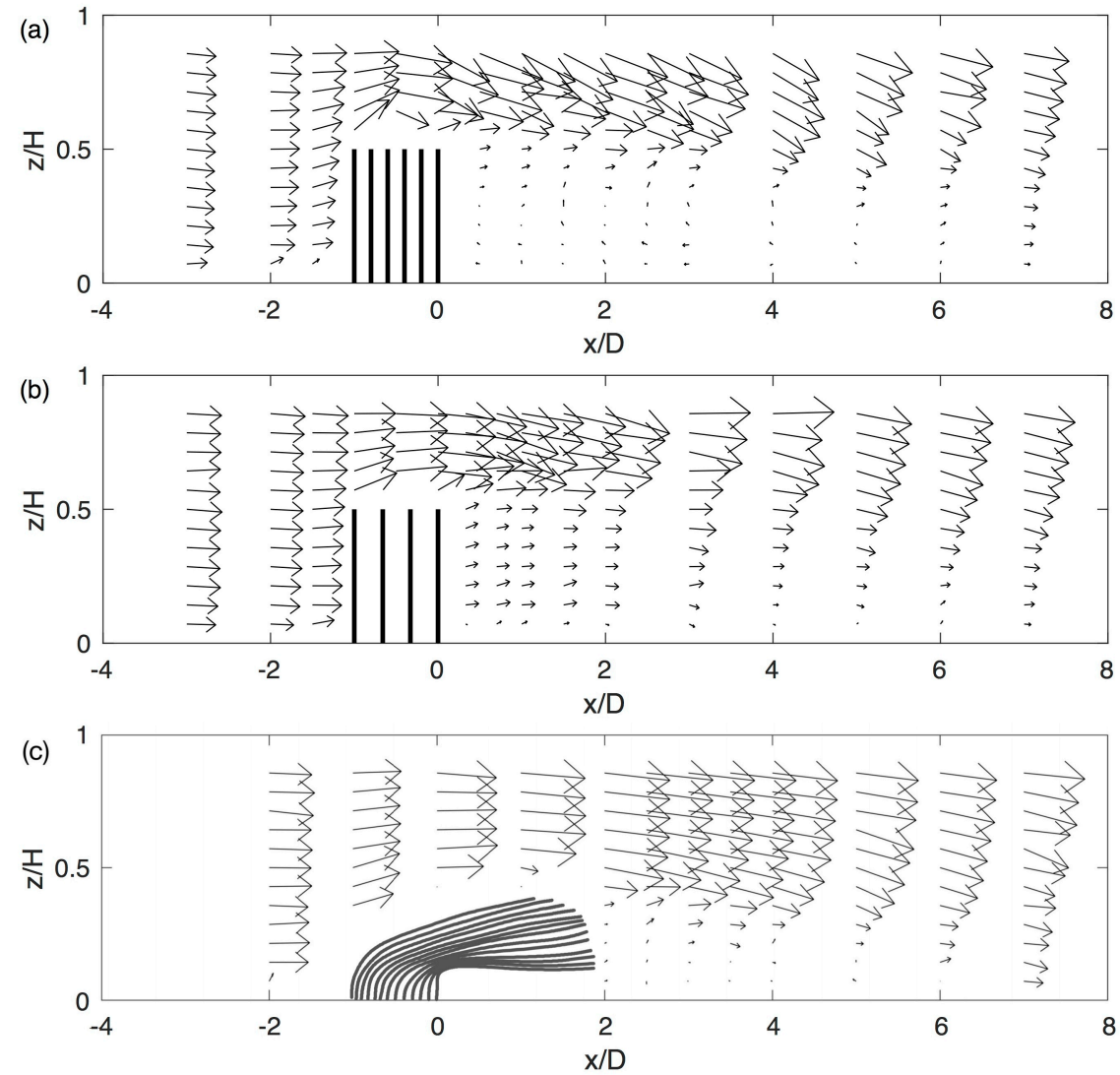

Figure 6. Velocity vectors around submerged, channel-spanning patches: (a) Case A1 ( $\phi=0.21)$, (b) Case A2 ( $\phi=0.15),(\mathrm{c})$ Case A5 ( $\phi=0.15)$. Heavy vertical lines in (a) and (b) represent the rigid stem patch. Curves in (c) represent the patch with flexible leaves. 


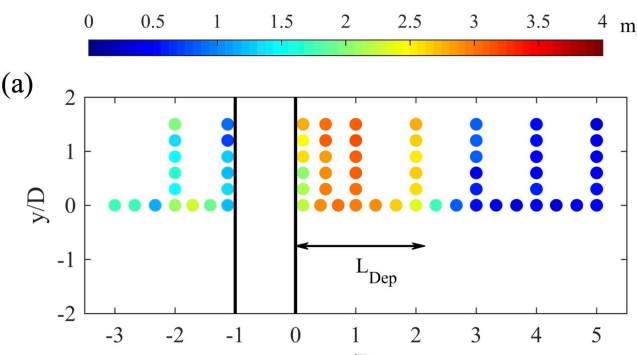

(b)

$\mathrm{x} / \mathrm{D}$

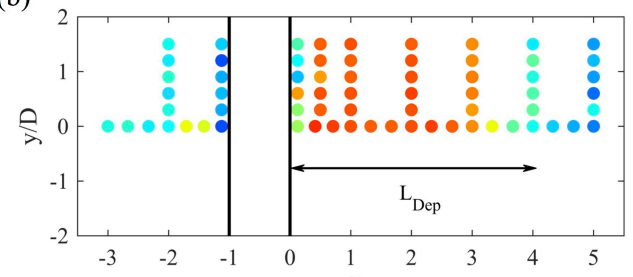

(c)

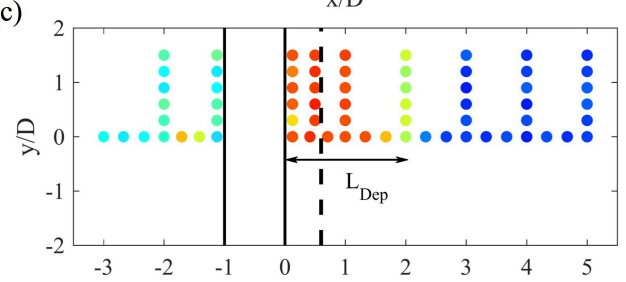

(d)

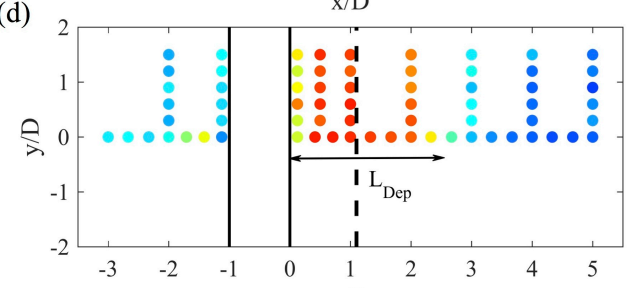

(e)

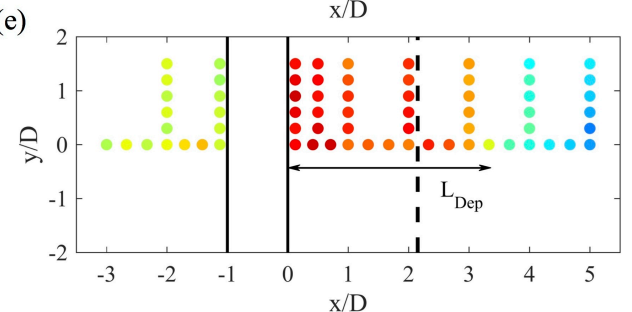

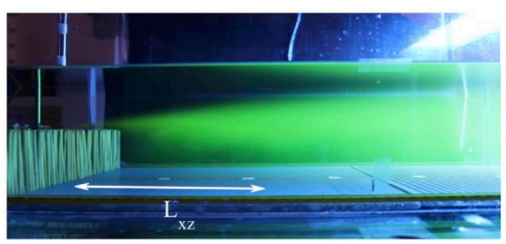

(g)

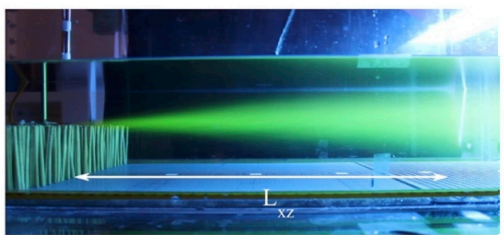

(h)

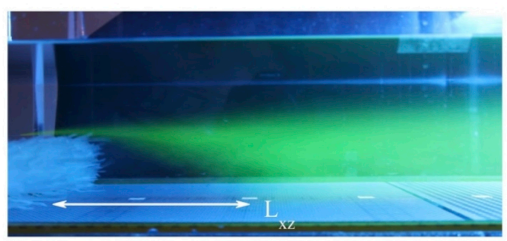

(i)

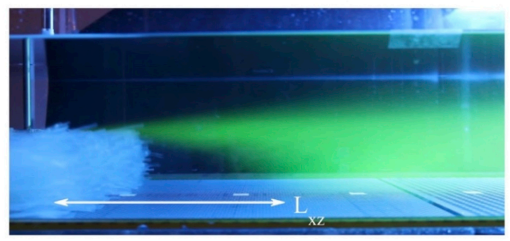

(j)

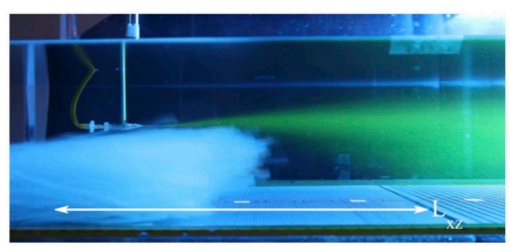

Figure 7. Spatial distribution of net deposition (left column) and time-averaged tracer visualization (right column) for submerged, rigid, channel-spanning patches and for submerged, channel-spanning patches with different flexible leave lengths, $L_{\text {leaf. }}$ (a) and (f) for Case A1 ( $\left.\phi=0.21\right)$; (b) and (g) for Case A2 $(\phi=0.15)$; (c) and (h) for Case A3 $L_{\text {leaf }}=6 \mathrm{~cm}$; (d) and (i) for Case A4 $L_{\text {leaf }}=12 \mathrm{~cm}$; (e) and (j) for Case A5 $L_{\text {leaf }}=26 \mathrm{~cm}$. The roots density of cases A3, A4 and A5 are equivalent to case A2, but with the addition of flexible leaves. However, the solid volume fraction of cases A3, A4 and A5 is similar to that of case A1. Left column (a) to (e): colored dots located at measurement positions, with color indicating magnitude of net deposition $\left(\mathrm{mg} / \mathrm{cm}^{2}\right)$, defined in color bar. The patch is located between $x / D=-1$ and 0 . Black dot-dash lines in (c), (d) and (e) indicate the trailing edge of the leaves. The double-headed arrow indicates the length of the enhanced deposition region $L_{D e p}$. Right column (f) and (j): Tracer released at top of patch. The region free of dye directly downstream of the patch corresponded to a region of low velocity and low turbulence. The distance, $L_{x z}$, was measured from the trailing edge of the patch to the leading edge of the $x-z$ plane eddy. 

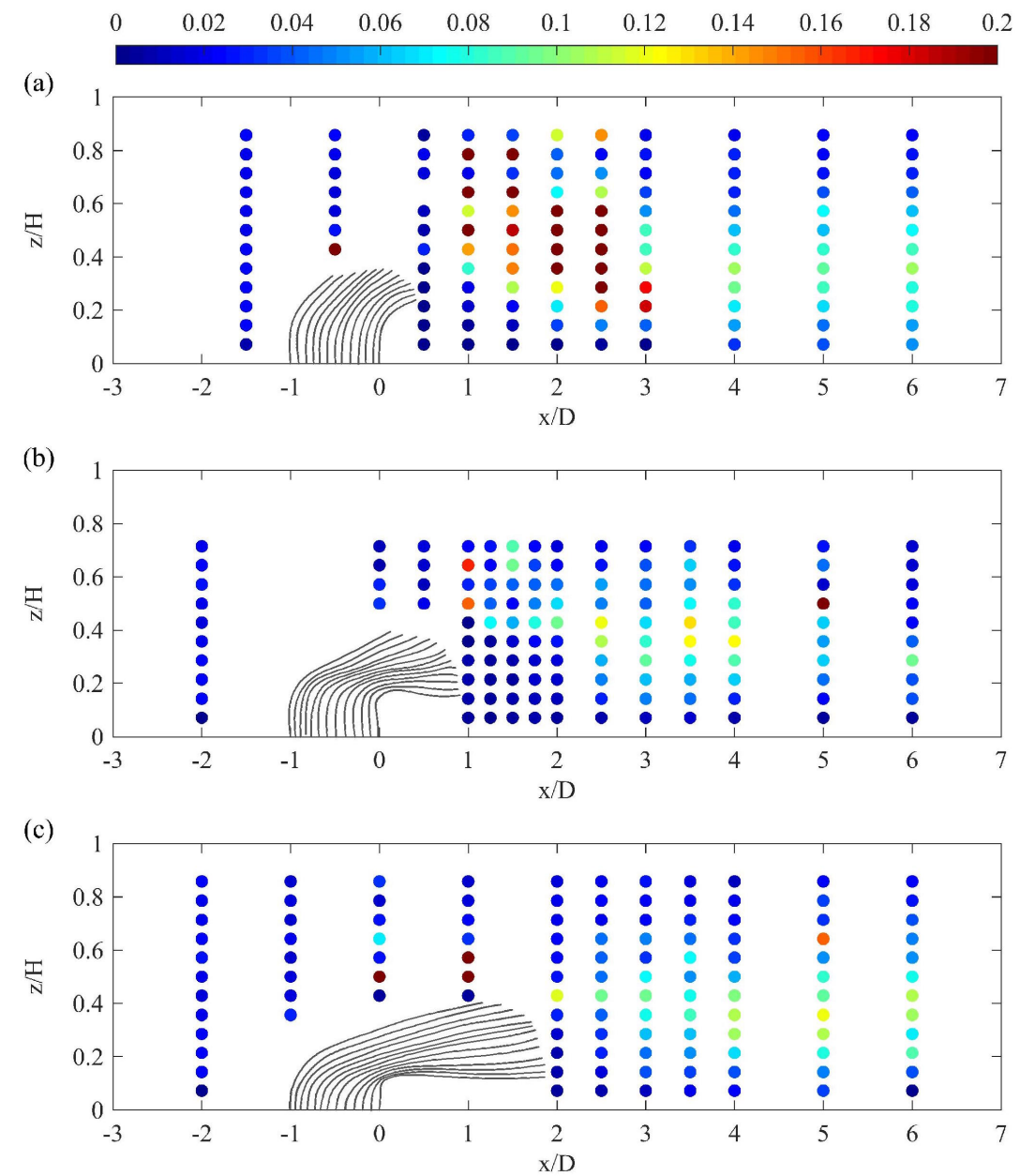

Figure 8. Distribution of normalized turbulent kinetic energy $\left(T K E / U_{0}^{2}\right)$ at the channel and patch centerline with $U_{0}$ $=9.1 \pm 0.1 \mathrm{~cm} / \mathrm{s}$. (a) Case A3 $L_{\text {leaf }}=6 \mathrm{~cm}$ (b) Case A4 $L_{\text {leaf }}=12 \mathrm{~cm}$, (c) Case A5 $L_{\text {leaf }}=26 \mathrm{~cm}$. The flow depth was $H$ $=14 \mathrm{~cm}$. The patch was located between $x / D=-1$ to 0 . 
$\begin{array}{lllllllll}0 & 0.5 & 1 & 1.5 & 2 & 2.5 & 3 & 3.5 & 4 \mathrm{mg} / \mathrm{cm}^{2}\end{array}$
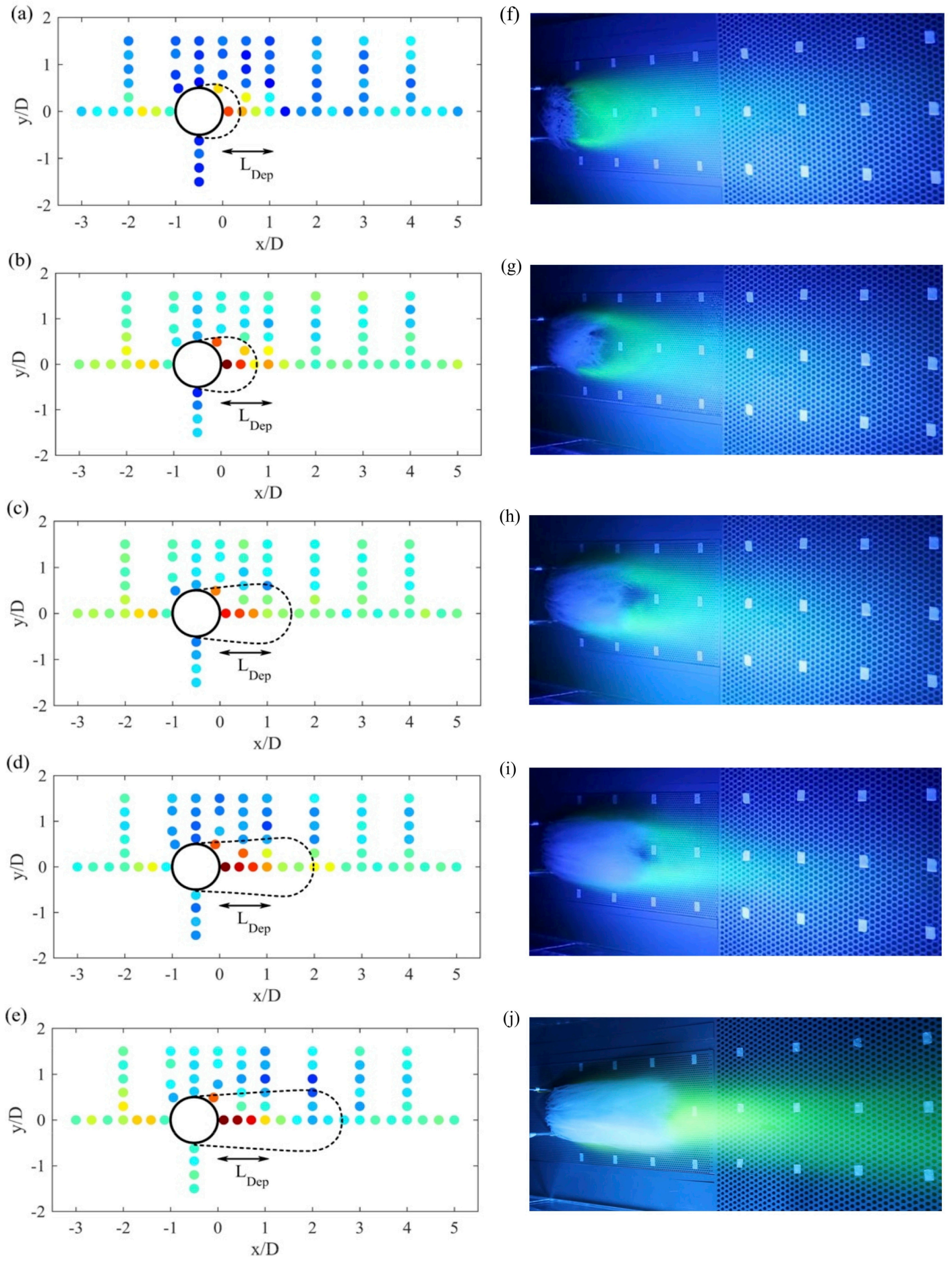

(j)

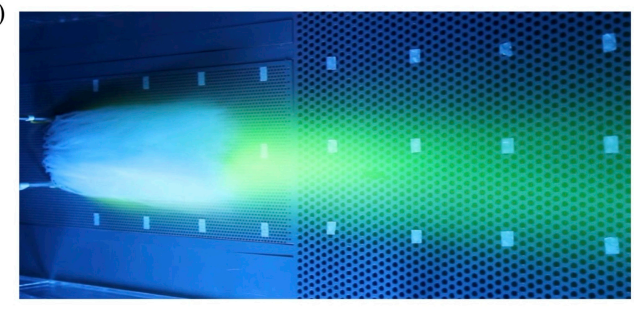

Figure 9. Spatial distribution of net deposition (left column) and time-averaged tracer visualization (right column) for circular patches with stem zone diameter $D=10 \mathrm{~cm}$ and the following leave lengths: (a) and (f) Case B1 $L_{\text {leaf }}=6 \mathrm{~cm}$; (b) and (g) Case B2 $L_{\text {leaf }}=12 \mathrm{~cm}$; (c) and (h) Case B3 $L_{\text {leaf }}=19 \mathrm{~cm}$; (d) and (i) Case B4 $L_{\text {leaf }}=26 \mathrm{~cm}$; (e) and (j) Case B5 $L_{\text {leaf }}=$ $33 \mathrm{~cm}$. Black circle represents the stem zone. The length of trailing leaves is shown with black dot-dash curve. 


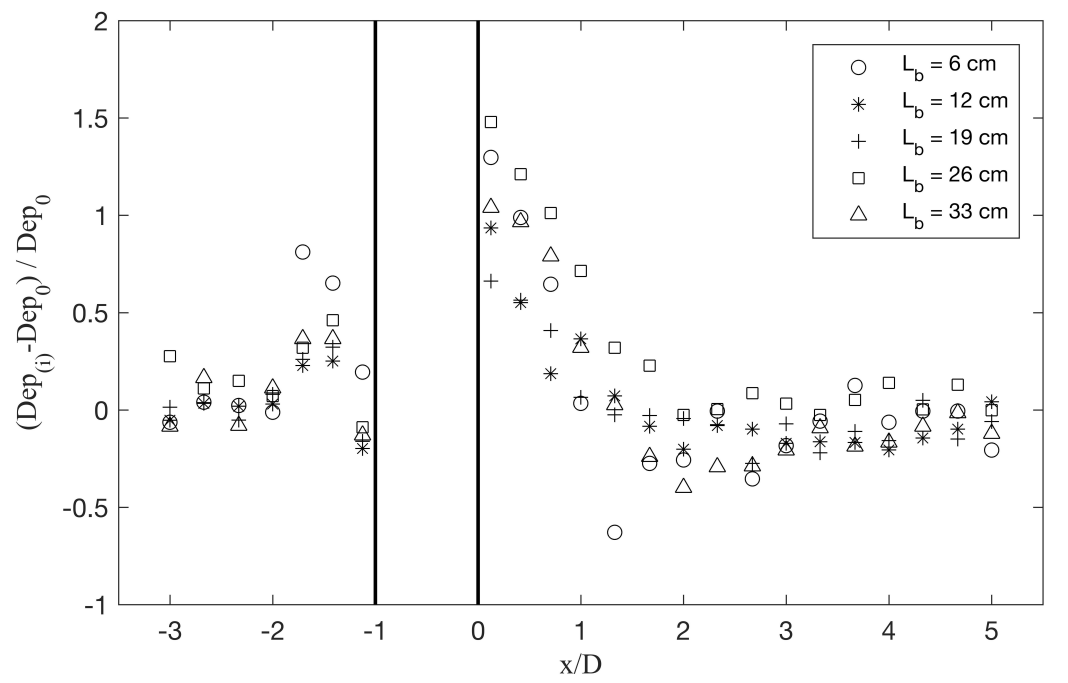

Figure 10. Longitudinal transects of normalized net deposition along the centerline of the submerged, circular patches with flexible leaves of different length (see legend). The net deposition at each point, $\operatorname{Dep}_{(i)}$, was normalized by the upstream deposition value, $D e p_{0}$. The vertical black lines indicate the stem region. 
(a) Time $t_{0}$

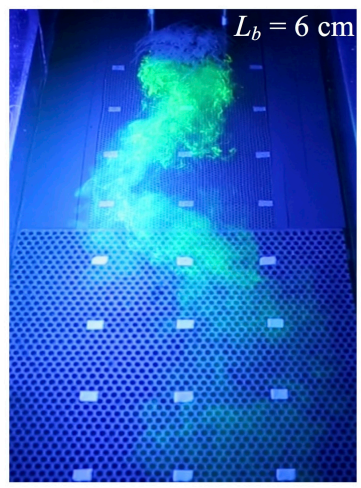

(b) Time $t_{0}+T_{k v} / 2$

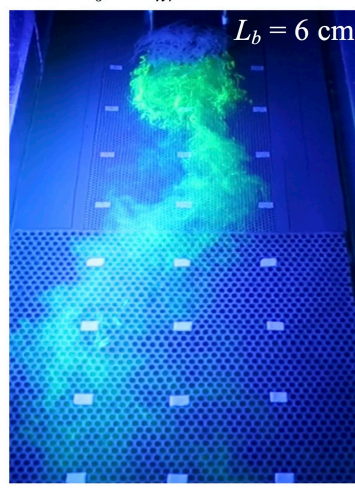

(c) $S_{v v}$

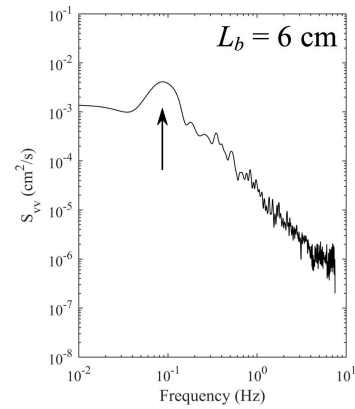

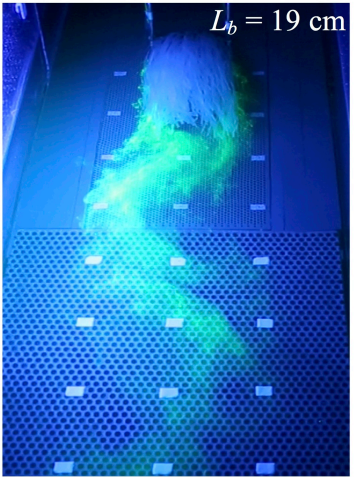
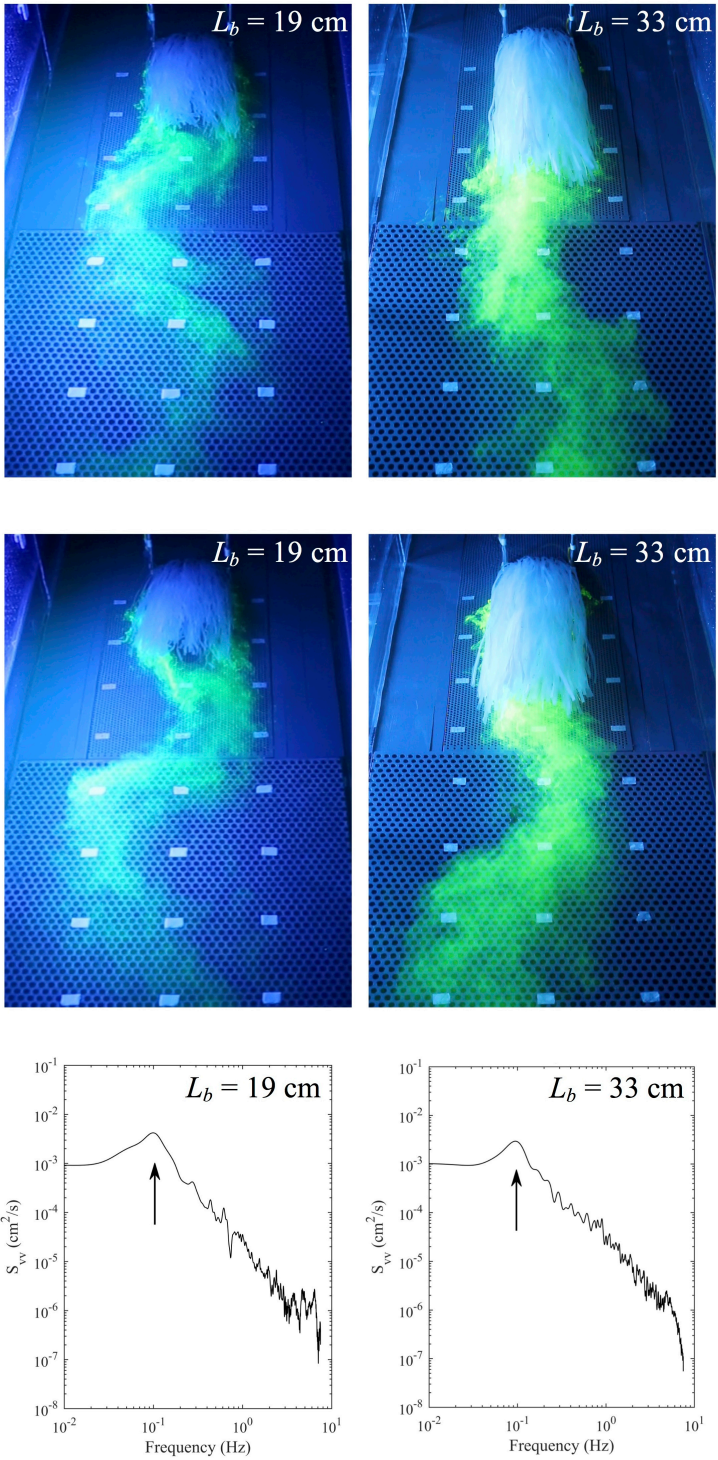

Figure 11. Top view of flow visualization experiment using UV light and fluorescein dye to visualize the wake downstream of a patch with diameter $D=10 \mathrm{~cm}$. Flow was from the top to bottom of the image. The dye was injected at the two outmost edges of the patch. The streamwise and lateral intervals between white markers on the channel bed were $10 \mathrm{~cm}$. (a) Images taken at time $t_{0}$; (b) Images taken at time $t_{0}+T_{k v} / 2$, with $T_{k v}$ the observed vortex street period; (c) Velocity spectrum $S_{v v}$ measured on the centerline $(y=0)$ directly behind patches. Different leave lengths $\left(L_{b}\right)$ were considered, e.g. $L_{\text {leaf }}=6(\mathrm{Case}$ B1), 19 (Case B3) and $33 \mathrm{~cm}$ (Case B5). The flow depth was $H=14 \mathrm{~cm}$. 


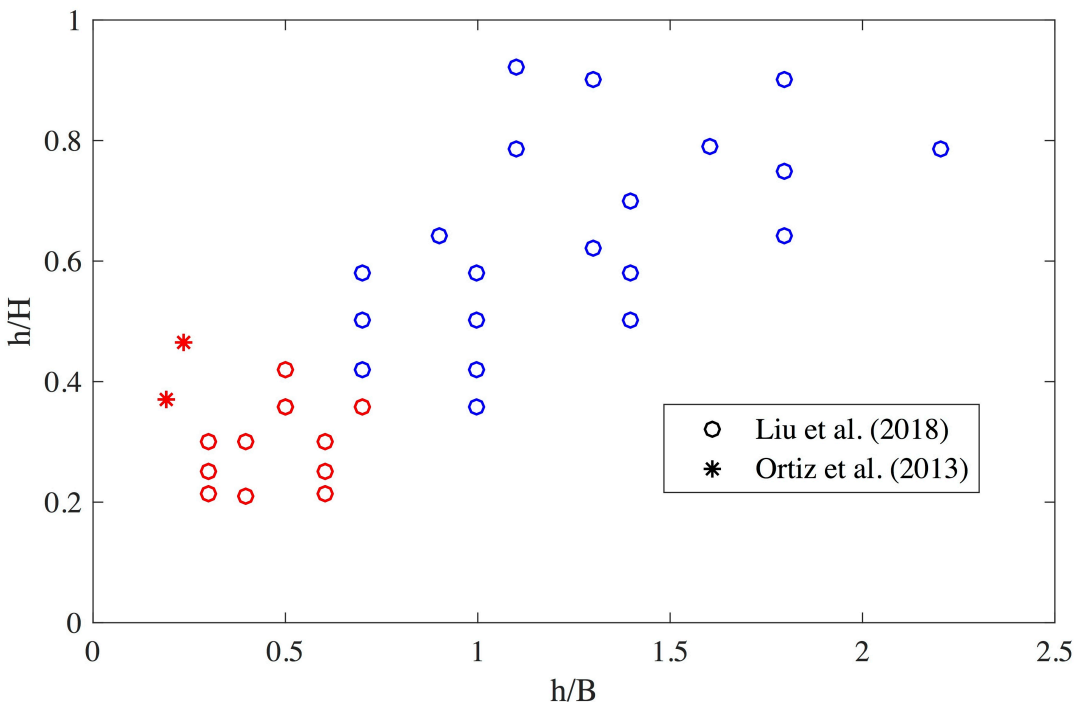

1

2 Figure 12. The absence (red symbols) or presence (blue symbols) of the von Karman vortex street as a 3 function of submergence ratio $(h / H)$ and height to width aspect ratio $(h / B)$. The data were taken from the $4 \quad$ Liu et al. 2018 and Ortiz et al. 2013.

5

6

7

8

9

10

11

12

13

14

15

16

17

18

19

20 


\section{References}

22 Bouma, T., L. Van Duren, S. Temmerman, T. Claverie, A. Blanco-Garcia, T. Ysebaert, and P. Herman (2007), Spatial flow and sedimentation patterns within patches of epibenthic structures: Combining field, flume and modelling experiments." Cont. Shelf Res., 27, 1020-1045, doi:10.1016/j.csr.2005.12.019.

Brookshire, E. and K. Dwire (2003), Controls on patterns of coarse organic particle retention in headwater streams. J. N. Am. Benth. Soc. 22, 17-34, doi:10.2307/1467975.

Castro, I. P. (1971), perforated plates normal to an air-stream. J. Fluid Mech, 46(part 3), 599-609, doi: $10.1017 / \mathrm{S} 0022112071000727$.

Chen, Z., A. Ortiz, L. Zong and H. Nepf (2012), The wake structure behind a porous obstruction and its implications for deposition near a finite patch of emergent vegetation. Water Res. Res. 48, W09517 12p., doi:10.1029/2012WR012224.

Chen, Z., C. Jiang, and H. Nepf (2013), Flow adjustment at the leading edge of a submerged aquatic canopy, Water Resour. Res., 49, 5537-5551, doi:10.1002/wrcr.20403.

Cotton, J., G. Wharton, J. Bass, C. Heppell, and R. Wotton (2006), The effects of seasonal changes to in-stream vegetation cover on patterns of flow and accumulation of sediment, Geomorphology, 77, 320-334, doi:10.1016/j.geomorph.2006.01.010

Chang, K., and Constantinescu, G. (2015). Numerical investigation of flow and turbulence structure through and around a circular array of rigid cylinders. Journal of Fluid Mechanics, 776, 161-199, doi:10.1017/jfm.2015.321.

Cheng, N. (1997). "Simplified settling velocity formula for sediment particle." J. Hydraulic Eng., 123, 149-152, doi:10.1061/(ASCE)0733-9429(1997)123:2(149).

Droppo, I., G. Leppard, D. Flannigan, and S. Liss (1997), The freshwater floc: A functional relationship of water and organic and inorganic floc constituents affecting suspended sediment properties, Water Air Soil Pollut., 99 (1-4), 43-54, doi:10.1023/A:1018359726978.

Droppo, I. (2004), Structural controls on floc strength and transport, Can. J. Civ. Eng., 31, 569-578, doi:10.1139/104-015.

Folkard, A. (2005), Hydrodynamics of model Posidonia oceanica patches in shallow water. Limnol. Ocean., 50, 1592-1600, doi:10.4319/lo.2005.50.5.1592.

Folkard, A. (2011), Flow regimes in gaps within stands of flexible vegetation: Laboratory flume simulations, Environ. Fluid Mech., 11(3), 289-306, doi:10.1007/s10652-010-9197-5.

Follett, E., and H. Nepf (2012), Sediment patterns near a model patch of reedy emergent vegetation, Geomorphology, 179, 141-151, doi:10.1016/j.geomorph.2012.08.006.

Ghisalberti, M. and H. Nepf. 2006. The structure of the shear layer over rigid and flexible canopies. Env. Fluid Mech., 6(3):277-301, doi:10.1007/s10652-006-0002-4.

Goring, D., and V. Nikora (2002), Despiking acoustic Doppler velo- cimeter data, J. Hydraul. Eng., 128(1),

58 Gurnell, A., W. Bertoldi, and D. Corenblit (2012), Changing river channels: The roles of hydrological 59 processes, plants and pioneer fluvial landforms in humid temperate, mixed load, gravel bed rivers, 
Earth-Science Rev., 111(1-2), 129-141, doi:10.1016/j.earscirev.2011.11.005.

Gurnell, A. (2014), Plants as river system engineers. Earth Surface Proc. and Landforms, 39, 4-25, doi:10.1002/esp.3397.

Hoover, T., L. Marczk, J. Richardson, and N. Yonemitsu (2010), Transport and settlement of organic matter in small streams, Freshwater Biol., 55(2), 436-449, doi:10.1111/j.1365-2427.2009.02292.

Jones, J., A. Collins, P. Naden, and D. Sear (2012), The relationship between fine sediment and macrophytes in rivers, River Res. Appl., 28(7), 1006-1018, doi:10.1002/rra.1486.

Huang, I., J. Rominger, H. Nepf. 2011. The motion of kelp leaves and the surface renewal model. Limnol. Ocean. 56(4): 1453-14562, doi:10.4319/1o.2011.56.4.1453.

Julien, P. Y. (1995), Erosion and sedimentation, Cambridge Univ. Press, New York, N. Y.

Kemp, J., D. Harper, and G. Crosa (2000), The habitat-scale ecohydraulics of rivers, Ecol. Eng., 16(1), 1729, doi:10.1016/S0925-8574(00)00073-2.

Kondziolka, J., and H. Nepf (2014), Vegetation wakes and wake interaction shaping aquatic landscape evolution. Limnol. and Ocean: Fluids Envir., 4: 1-14, doi:10.1215/21573689-2846314.

Larsen, L. G., Harvey, J. W., Noe, G. B., and Crimaldi, J. P. (2009). Predicting organic floc transport dynamics in shallow aquatic ecosystems: Insights from the field, the laboratory, and numerical modeling. Water Resources Research, 45(1), doi: 10.1029/2008WR007221.

Lima, P., J. Janzen and H. Nepf (2015), Flow patterns around two neighboring patches of emergent vegetation and possible implications for deposition and vegetation growth. Env. Fluid Mech., doi:10.1007/s10652-015-9395-2.

Liu, C. and H. Nepf (2016), Sediment deposition within and around a finite patch of model vegetation over a range of channel velocity. Water Res. Res. 51(1): 600-612, doi:10.1002/2015WR018249.

Liu, C. Hu, Z. Lei, J. H. Nepf (2018), Vortex structure and sediment deposition in the wake behind a finite patch of model submerged vegetation. Journal of Hydraulic Engineering, 144(2): 04017065, doi: 10.1061/(ASCE)HY.1943-7900.0001408.

Nicolle, A. and Eames, I. (2011). Numerical study of flow through and around a circular array of cylinders. Journal of Fluid Mechanics, 679, 1-31, doi:10.1017/jfm.2011.77.

Ortiz, A., A. Ashton, and H. Nepf (2013), Mean and turbulent velocity field near rigid and flexible plants, and the implications for deposition. J. Geophys. Res.- Earth Surface, 118, 1-15, doi:10.1002/2013JF002858.

Perera, M. 1981. Shelter behind two-dimensional solid and porous fences. J. Wind Eng. and Industrial Aero., 8, 93-104, doi:10.1016/0167-6105(81)90010-6.

Plew, D., G. Cooper, and F. Callaghan (2008), Turbulence-induced forces in a freshwater macrophyte canopy. Water Res. Res., 44, W02414, doi:10.1029/2007WR006064.

Rominger, J., A. Lightbody, and H. Nepf (2010), Effects of added vegetation on sand bar stability and stream hydrodynamics, J. Hydraul. Eng., 136(12), 994-1002, doi:10.1061/(asce)hy.1943-7900.0000215. kelp leaves. Limnol. Ocean., 59(6), 2028-2041, doi:10.4319/1o.2014.59.6.2028. 
99 Sand-Jensen, K., and T. Madsen (1992), Patch dynamics of the stream macrophyte, Callitriche cophocarpa. Freshwater Biol., 27:277-282, doi: 10.1111/j.1365-2427.1992.tb00539.x. in lowland streams. Freshwater Biol 39: 663-6, doi: 10.1046/j.1365-2427.1998.00316.x.

Sand-Jensen, K., and M. Pedersen (2008), Streamlining of plant patches in streams, Freshwater Biol., 53(4), 714-726, doi:10.1111/j.1365-2427.2007.01928.x.

Schulz, M., H. Kozerski, T. Pluntke, and K. Rinke (2003), The influence of macrophytes on sedimentation and nutrient retention in the lower River Spree, Water Res., 37, 569-578, doi:10.1016/S0043-1354(02)00276-2.

Shi, Y., B. Jiang, and H. Nepf (2016), Influence of particle size and density, and channel velocity on the deposition patterns around a circular patch of model vegetation. Water Res. Res 52, 1044-1055, doi:10.1002/2015WR018278.

Siniscalchi, F., and V.Nikora (2012), Flow-plant interactions in open-channel flows: A comparative analysis of five freshwater plant species. Water Res. Res., 49, W05503, doi:10.1029/2011WR011557.

Statzner, B., and R. Mueller (1989), Standard hemispheres as indicators of flow characteristics in lotic benthos research, Freshwater Biol., 21(3), 445-459, doi:10.1111/j.1365-2427.1989.tb01377.x.

Taddei, S., Manes, C., and Ganapathisubramani, B. (2016). Characterisation of drag and wake properties of canopy patches immersed in turbulent boundary layers. Journal of Fluid Mechanics, 798, 27-49, doi.org/10.1017/jfm.2016.312.

Tanaka, N., and J. Yagisawa (2010), Flow structures and sedimentation characteristics around clump-type vegetation, J. Hydro-Environment Res., 4(1), 15-25, doi:10.1016/j.jher.2009.11.002.

White, B., and H. Nepf (2007), Shear instability and coherent structures in shallow flow adjacent to a porous layer. J. Fluid Mech., 593, 1-32, doi:10.1017/S0022112007008415.

Zong, L., and H. Nepf. (2011), Vortex development behind a finite porous obstruction in a channel., $J$. Fluid Mech., 691: 368-391, doi:10.1017/jfm.2011.479. 